\title{
Detection and Characterization of Nano-Defects Located on Micro-Structured Substrates by Means of Light Scattering
}

\author{
Pablo Albella, ${ }^{1}$ Francisco González, ${ }^{1}$ Fernando Moreno, ${ }^{1}$ \\ José María Saiz ${ }^{1}$ and Gorden Videen ${ }^{2}$ \\ ${ }^{1}$ University of Cantabria \\ ${ }^{2}$ Army Research Laboratory \\ ${ }^{1}$ Spain \\ ${ }^{2} U S A$
}

\section{Introduction}

Detection and characterization of microstructures is important in many research fields such as metrology, biology, astronomy, atmospheric contamination, etc. These structures include micro/nano particles deposited on surfaces or embedded in different media and their presence is typical, for instance, as a defect in the semiconductor industry or on optical surfaces. They also contribute to SERS and may contribute to solar cell performance [Sonnichsen et al., 2005; Stuart et al., 2005; Lee et al., 2007]. The central problem related to the study of morphological properties of microstructures (size, shape, composition, density, volume, etc.) is often lumped into the category of "Particle Sizing" and has been a primary research topic [Peña et al., 1999; Moreno and Gonzalez, 2000; Stuart et al., 2005; Lee et al., 2007].

There are a great variety of techniques available for the study of micro- and nanostructures, including profilometry and microscopy of any type: optical, electron, atomic force microscopy (AFM), etc. Those based on the analysis of the scattered light have become widely recognized as a powerful tool for the inspection of optical and non-optical surfaces, components, and systems. Light-scattering methods are fast, flexible and robust. Even more important, they are generally less expensive and non-invasive; that is, they do not require altering or destroying the sample under study [Germer et al., 2005; Johnson et al., 2002; Mulholland et al., 2003].

In this chapter we will focus on contaminated surfaces composed of scattering objects on or above smooth, flat substrates. When a scattering system gets altered either by the presence of a defect or by any kind of irregularity on its surface, the scattering pattern changes in a way that depends on the shape, size and material of the defect. Here, the interest lies not only in the characterization of the defect (shape, size, composition, etc.), but also on the mere detection of its presence. We will show in detail how the analysis of the backscattering patterns produced by such systems can be used in their characterization. This may be useful in practical situations, like the fabrication of a chip in the semiconductor industry in the case of serial-made microstructures, the performance of solar cells, for detection and 
characterization of contaminants in optical surfaces like telescope mirrors or other sophisticated optics, and for assessing surface roughness, etc. [Liswith, 1996; Chen, 2003]. Before considering the first practical situation, we find it convenient to describe the backscattering detection concept.

\section{Backscattering detection}

In a typical scattering experiment, a beam of radiation is sent onto a target and the properties of the scattered radiation are detected. Information about the target is then extracted from the scattered radiation. All situations considered in this work exploit this detection scenario in the backscattering direction. Although backscattered light may be the only possible measurement that can be made in some situations, especially when samples are crowded with other apparati, it also does have some advantages that make it a useful approach in other situations. Backscattering detection can be very sensitive to small variations in the geometry and/or optical properties of scattering systems with structures comparable to the incident wavelength. It will be shown how an integration of these, over either the positive or negative quadrant, corresponding to the defect side or the opposite one, respectively, yields a parameter that allows one not only to deduce the existence of a defect, but also to provide some information about its size and location on the surface, constituting a non-invasive method for detecting irregularities in different scattering systems.

\section{System description}

Figure 1 shows an example of a typical practical situation of a microstructure that may or may not contain defects. In this case, the microstructure is an infinitely long cylinder, or fiber. Together with the real sample, we show the 2D modelling we use to simulate this situation and provide a 3D interpretation.

This basic design consists on an infinitely long metallic cylinder of diameter $D$, placed on a flat substrate. We define two configurations: the Non-Perturbed Cylinder (NPC) configuration, where the cylinder has no defect and the Perturbed Cylinder (PC) configuration, which is a replica of the NPC except for a defect that can be either metallic or dielectric and can be located either on the cylindrical microstructure itself or at its side, lying on the flat substrate underneath. We consider the spatial profile of this defect to be cylindrical, but other defect shapes can be considered without difficulty. The cylinder axis is parallel to the $\mathrm{Y}$ direction and the $\mathrm{X}-\mathrm{Z}$ plane corresponds to both the incidence and scattering planes. This restricts the geometry to the two-dimensional case, which is adequate for the purpose of our study [Valle et al., 1994; Moreno et al., 2006; Albella et al.,2006; Albella et al., 2007]. The scattering system is illuminated by a monochromatic Gaussian beam of wavelength $\lambda(633 \mathrm{~nm})$ and width $2 \omega_{0}$, linearly polarized perpendicular to the plane of incidence (S-polarized).

In order to account for the modifications introduced by the presence of a defect in the scattering patterns of the whole system, we use the Extinction theorem, which is one of the bases of modern theories developed for solving Maxwell's Equations. The primary reason for this choice is that it has been proven a reliable and effective method for solving 2D lightscattering problems of rounded particles in close proximity to many kinds of substrates [Nieto-Vesperinas et al., 1992; Sanchez-Gil et al., 1992; Ripoll et al., 1997; Saiz et al., 1996]. 


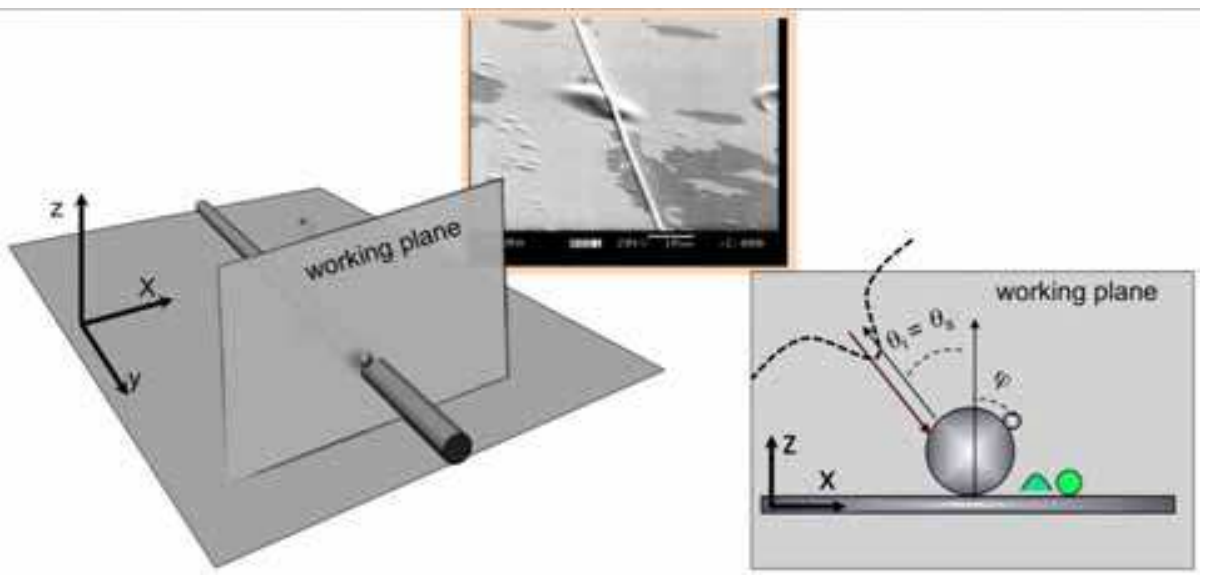

Fig. 1. Example of a contaminated microstructure (top figure) and its corresponding $2 \mathrm{D}$ and 3D models.

The Extinction theorem is a numerical algorithm. To perform the calculations, it is necessary to discretize the entire surface contour profile (substrate, cylinder and defect) into an array of segments whose length is much smaller than any other length scale of the system, including the wavelength of light and the defect. Bear in mind that it is important to have a partition fine enough to assure a good resolution in the high curvature regions of the surface containing the lower portion of the cylinder and defect. Furthermore, and due to obvious computing limitations, the surface has to be finite and the incident Gaussian beam has to be wide enough to guarantee homogeneity in the incident beam but not so wide as to produce undesirable edge effects at the end of the flat surface. Consequently, in our calculations, the length of the substrate has been fixed to $80 \lambda$ and the width $\left(2 \omega_{0}\right)$ of the Gaussian beam to $8 \lambda$.

\section{Metallic substrates}

In this section we initially discuss the case of metallic cylinders, or fibers, deposited on metallic substrates and with the defect either on the cylinder itself or on the substrate but near the cylinder.

\section{Defect on the Cylinder}

As a first practical situation, Figure 2 shows the backscattered intensity pattern, as a function of the incident angle $\theta_{i}$, for a metallic cylinder of diameter $D=2 \lambda$. We consider two different types of defect materials of either silver or glass and having diameter $d=0.15 \lambda$.

It can be seen how the backscattering patterns measured on the unperturbed side of the cylinder (corresponding to $\theta_{s}<0$ ) remain almost unchanged from the reference pattern. In this case, we could say that the defect was hidden or shadowed by the incident beam. If the scattering angle is such that the light illuminates the defect directly, a noticeable change in the positions and intensity values of the maxima and minima results. The number of maxima and minima observed may even change if the defect is larger than $0.4 \lambda$. This means that there is no change in the effective size of the cylinder due to the presence of the defect. This result can be explained using a phase-difference model [Nahm \& Wolf, 1987; Albella et al., 2007], where the substrate is replaced by an image cylinder located opposite the 
substrate from the real one. Then, we can consider the two cylinders as two coherent scatterers. The resultant backscattered field is the linear superposition of the scattered fields from each cylinder, which only differ by a phase corresponding to the difference in their optical paths and reflectance shifts. This phase difference is directly related to the diameter of the cylinder. See the Appendix for more detail on this model.
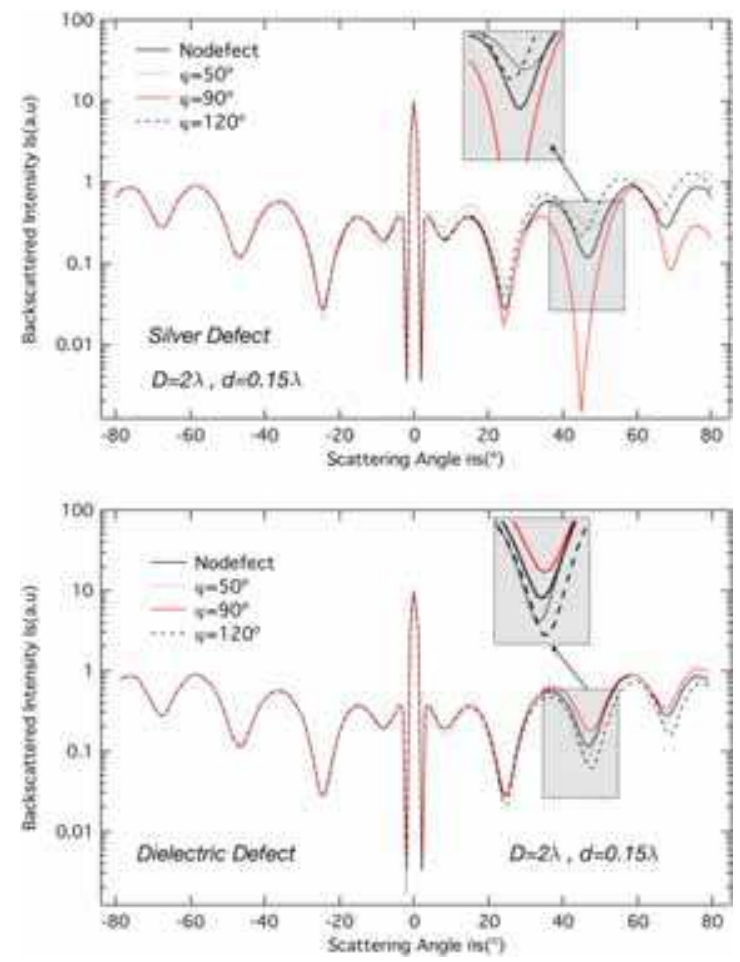

Fig. 2. Backscattered intensity pattern $I_{b a c k}$ as a function of the incident angle $\theta_{i}$ for a metallic cylinder of diameter $D=2 \lambda$. Two different types of defect material (either silver or glass) have been considered, with a diameter $d=0.15 \lambda$ [Albella et al., 2007].

One other interesting point is that the backscattering pattern has nearly the same shape in terms of intensity values and minima positions, regardless of the nature of the defect. Perhaps, the small differences observed manifest themselves better when the defect is metallic. However for $\theta_{s}>0$, a difference in the backscattered intensity can be noticed when comparing the results for silver and glass defects. If we observe a minimum in close detail (magnified regions), we see how $I_{\text {back }}$ increases with respect to the cylinder without the defect when it is made of silver. In the case of glass located at the same position, $I_{\text {back }}$ decreases. These differences can be analyzed by considering an incremental integrated backscattering parameter $\sigma_{b r}$, which is the topic of the next section.

\section{Parameter $\sigma_{b r}$}

One of the objectives outlined in the introduction of this chapter was to show how the backscattering pattern changes when the size and the position of a defect are changed, and 
whether it is possible to find a relationship between those changes and the defect properties of size and position. A systematic analysis of the pattern evolution is necessary. The possibility of using the shift in the minima to obtain the required information [Peña et al., 1999] is not suitable in this case because there is no consistency in the behaviour of the angular positions of the minima with defect change. Based on the loss of symmetry in the backscattering patterns introduced by the cylinder defect, a more suitable parameter can be introduced to account for these variations. We have defined it as

$$
{\sigma_{b r}}^{ \pm}=\frac{{\sigma_{b}{ }^{ \pm}-\sigma_{b 0}{ }^{ \pm}}_{\sigma_{b 0}{ }^{ \pm}}}{\sigma_{b 0}{ }^{ \pm}}-1
$$

where

$$
\sigma_{b}^{ \pm}=\int_{0}^{ \pm 90^{\circ}} I_{b a c k}\left(\theta_{s}\right) \cdot d \theta_{s}
$$

is the backscattering intensity $\left(I_{\text {back }}\right)$ integrated over either the positive $\left(0^{\circ}\right.$ to $\left.90^{\circ}\right)$ or the negative $\left(0^{\circ}\right.$ to $\left.-90^{\circ}\right)$ quadrant. Subscript 0 stands for the NPC configuration. One of the reasons for using $\sigma_{b r} \pm$ is that integrating over $\theta_{s}$ allows us to account for changes produced by the defect in the backscattering efficiencies associated with an entire backscattering quadrant, not just in a fixed direction.

Figure 3 shows a comparison of $\sigma_{b r}$ calculated from the scattering patterns shown in Figure 2 , as a function of the angular position of the defect on the main cylinder. It can be seen that the maximum value of $\sigma_{b r} \pm$ has an approximate linear dependence on the defect size $d$. As an example, for the case of a metallic defect near a $D=2 \lambda$ cylinder, $\left[\sigma_{b r}\right]_{\max }=2.51 d-0.14$ with a regression coefficient of 0.99 and $d$ expressed in units of $\lambda$. For $d \in[0.05 \lambda, 0.2 \lambda]$ and cylinder sizes comparable to $\lambda$, it is found that the positions $\left[\sigma_{b r}\right]_{\max }$ and $\left[\sigma_{b r}\right]_{\min }$ are independent of the cylinder size $D$. Another characteristic of the evolution of $\sigma_{b r}{ }^{+}$is the presence of a minimum or a maximum around $\varphi=90^{\circ}$ for metallic and dielectric defects, respectively. Examining the behaviour of this minimum allows us to conclude that $\left[\sigma_{b r}\right]_{\min }$ also changes linearly with defect size; however, the slope is no longer independent of the cylinder size.

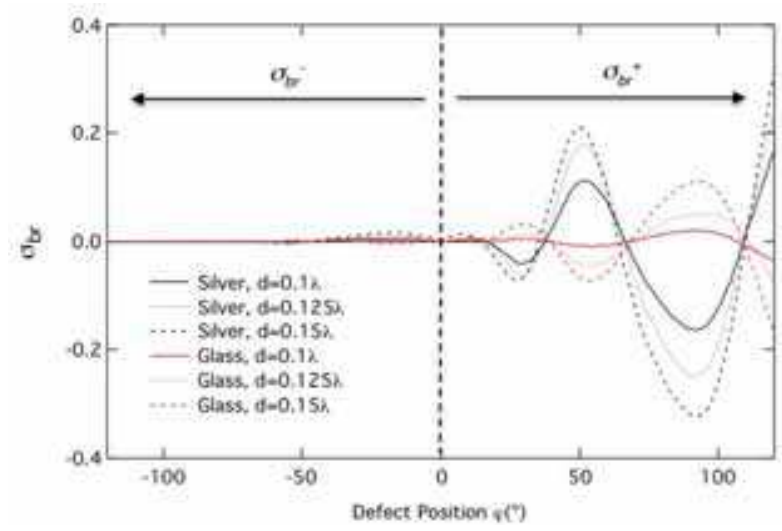

Fig. 3. $\sigma_{b r} \pm$ comparison for two different types of defects as a function of the defect position for a silver cylinder of $D=2 \lambda$ [Albella et al., 2007]. 
The most interesting feature shown in Figure 3 is that in all cases considered, $\sigma_{b r}$ for a glass defect has the opposite behaviour of that observed for a silver defect. That is, when the behaviour of the dielectric is maximal, the behaviour of the conductor is minimal, and vice versa. This behaviour suggests a way to discriminate metallic from dielectric defects. We shall focus now on the evolution of parameter $\sigma^{ \pm}$with the optical properties of the defect and in particular for a dielectric defect around the regions where the oscillating behaviour of $\sigma_{b r}$ reaches the maximum amplitude, that is, $\varphi=50^{\circ}$ and $\varphi=90^{\circ}$.

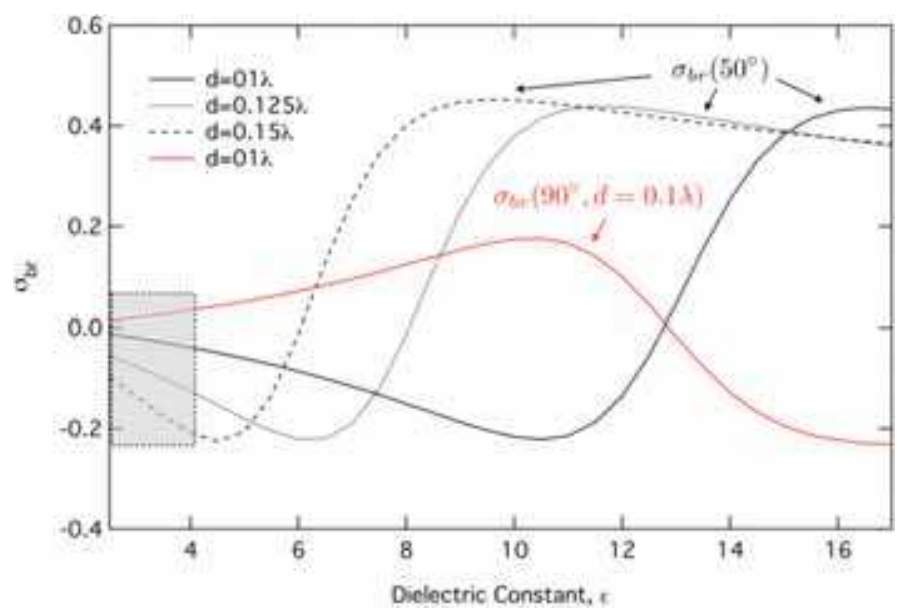

Fig. 4. Evolution of $\sigma_{b r^{ \pm}}$with $\varepsilon$ for a fixed defect position $\left(50^{\circ}\right)$. The behaviour for the glass defect (red) is opposite that of the silver defect (black) [Albella et al., 2007].

Figure 4, shows three curves of $\sigma_{b r}{ }^{+}\left(50^{\circ}\right)$ as a function of the dielectric constant $\varepsilon$ (ranging from 2.5 to 17), for three different defect sizes. For each defect size, $\sigma_{b r}{ }^{+}\left(50^{\circ}\right)$ begins negative and with negative slope; it reaches a minimum $(-0.22)$ and then undergoes a transition to a positive slope to a maximum (approximately 0.45 ). This means that for each size, there is a value of $\varepsilon$ large enough to produce values of $\sigma_{b r}{ }^{+}\left(50^{\circ}\right)$ similar to those obtained for silver defects. The zero value would correspond to a situation where $\sigma_{b r}{ }^{+}\left(50^{\circ}\right)$ cannot be used to discriminate the original defect. When the former analysis is repeated for $\varphi=90^{\circ}$ similar behaviour is found, although $\sigma_{b r}{ }^{+}\left(90^{\circ}\right)$ has the opposite sign, as expected. As an example, $\sigma_{b r}{ }^{+}\left(90^{\circ}\right)$ for a $d=0.1 \lambda$ defect is shown in Figure 4. Analogue calculations have been carried out for different values of $D$ ranging from $\lambda$ to $2 \lambda$, leading to similar results, i.e., the same $\sigma_{b r}(\varepsilon)$ with zero values is obtained for different values of $\varepsilon$. The region shadowed in Figure 4, typically a glass defect, can be fit linearly and could produce a direct estimation of the dielectric constant of the defect. As an example, $\sigma_{b r}\left(50^{\circ}\right)=-0.03 \varepsilon+0.02$ for the case of $d=0.1 \lambda$.

\section{Defect on the substrate}

We now consider the defect located on the substrate close to the main cylinder, within 1 or 2 wavelengths. In Figure 5 we see that there remains a clear difference in the backscattering patterns obtained in each of the two hemispheres, thus making it possible to predict which side of the cylinder the defect is located. Results shown in Figure 4 correspond to a cylinder of $D=2 \lambda$ and defect positions: $x=1.2 \lambda, 2 \lambda$, while the defect size is fixed at $d=0.2 \lambda$. 


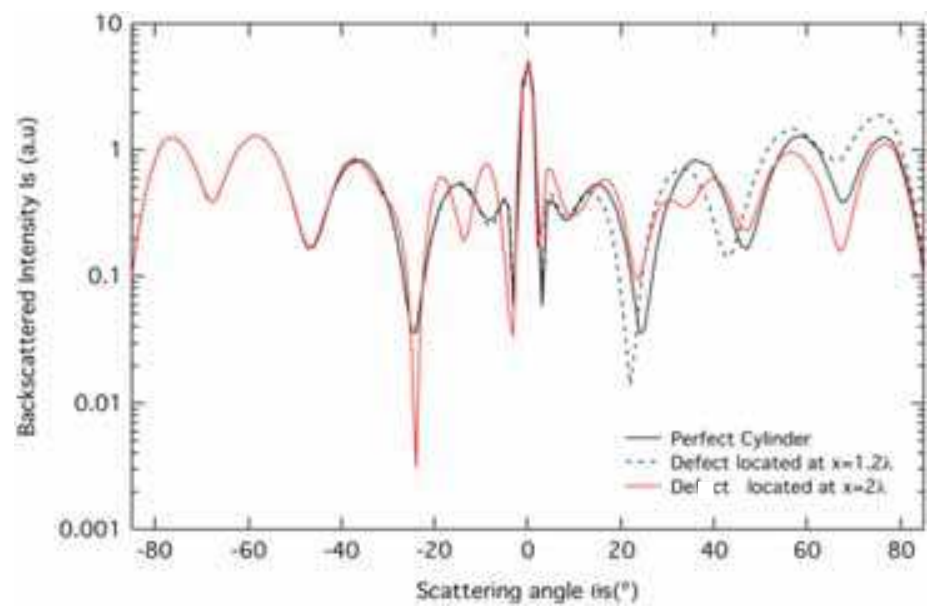

Fig. 5. Backscattering patterns obtained for when the defect is located on the substrate, together with the perfect, isolated cylinder case.

We observe again that the backscattering pattern changes more on the side where the defect is located. This behaviour is similar to that observed in the case of a defect on the cylinder. However, if we look at the side opposite the defect, the backscattering pattern does change if the defect is located outside the shadow cast by the cylinder. When the defect is near the cylinder $(x=1.2 \lambda)$, that is, within the shadow region, the change in the scattering pattern is negligible at any incident angle. Nevertheless, when the defect is located further from the cylinder $(x=2 \lambda)$, the change in the left-hand side can be noticed for incidences as large as $\theta_{i}=40^{\circ}$.

Figure 6 shows the evolution of $\sigma_{b r}$ for two different cylinders of $D=1 \lambda$ and $D=2 \lambda$, and for three different sized defects, $d=0.1 \lambda, 0.15 \lambda$ and $0.2 \lambda$. The shadowed area represents defect positions beneath the cylinder, not considered in the calculations. The smaller shadow produced in the $D=\lambda$ case causes oscillations in $\sigma_{b r}{ }^{-}$for smaller values of $x$.

It is worth noting that for a given cylinder size, the presence and location of a defect can be monitored. For a $D=\lambda$ cylinder with $x$ as great as $2 \lambda, \sigma_{b r}{ }^{-}$can increase as much as $10 \%$ for a defect of $d=0.2 \lambda$. When the defect is closer than $x=1.5 \lambda, \sigma_{b r}{ }^{+}$becomes negative while $\sigma_{b r}{ }^{-}$is not significant. Finally, when $x<\lambda, \sigma_{b r}{ }^{+}$is very sensitive and strongly tends to zero. In the case of a $D=2 \lambda$ cylinder, the most interesting feature is the combination of high absolute values and the strong oscillation of $\sigma_{b r}{ }^{+}$for $x$ within the interval $[\lambda, 2 \lambda]$. Here the absolute value of $\left|\sigma_{b r}{ }^{+}\right|$indicates the proximity of the defect, and the sign designates the location within the interval.

Although the size of the defect does not change the general behaviour, it is interesting to notice that when comparing both cases, $\sigma_{b r}{ }^{+}$is sensitive to the defect size and also dependent on the size of the cylinder, something that did not occur in the former configuration when the defect was located on the cylinder. Both situations can be considered as intrinsically different scattering problems: with the defect on the substrate, there are two distinct scattering particles, but with the defect on the cylinder, the defect is only modifying slightly the shape of the cylinder and consequently the overall scattering pattern.

To illustrate this difference, Figure 7 shows some examples of the near-field and far-field patterns produced by both situations for different defect positions. Figure 7(a) shows the 

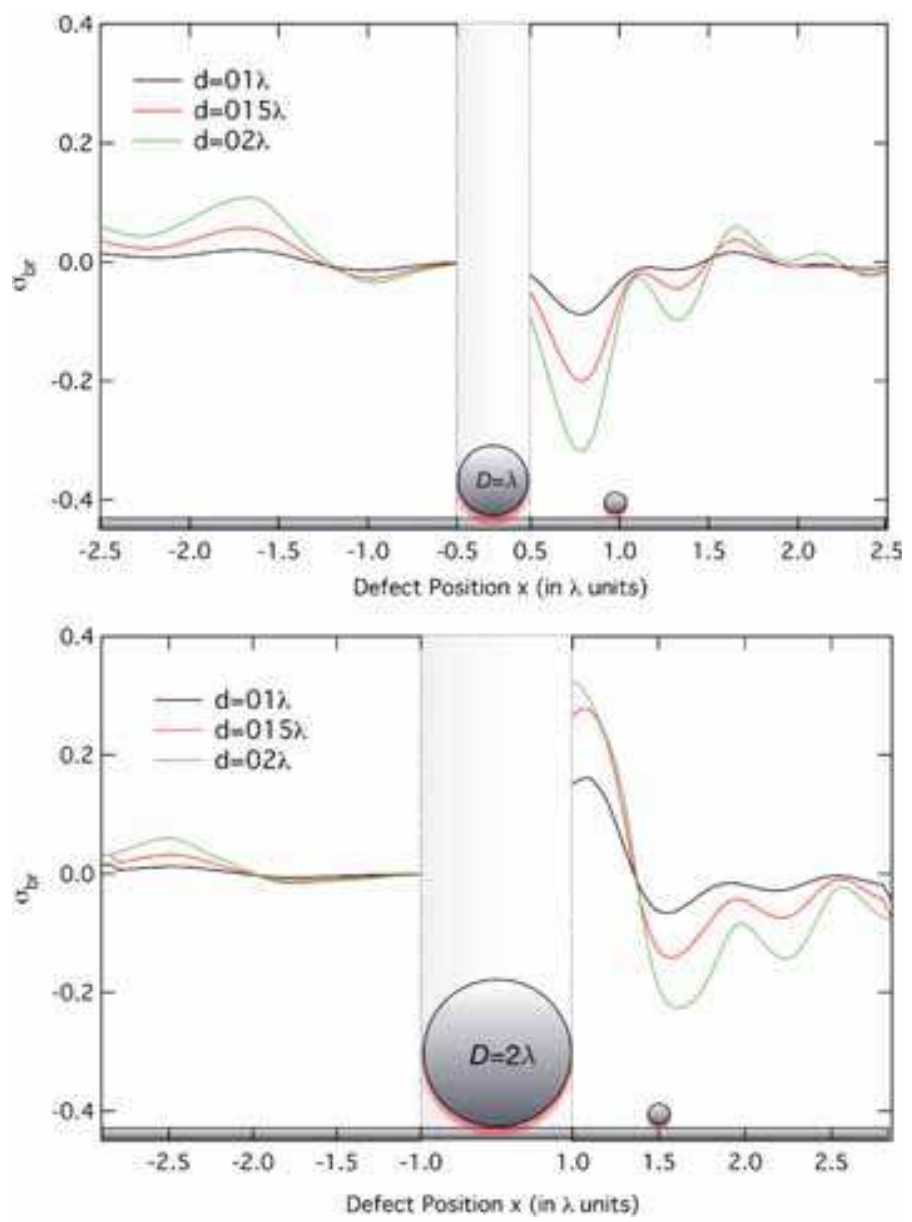

Fig. 6. $\sigma_{b r}$ for two different cylinders sizes, $D=\lambda, 2 \lambda$ and three defect sizes $d=0.1 \lambda, 0.15 \lambda$ and $0.2 \lambda$. Defect position $x$ ranges from $0.5 \lambda$ to $3 \lambda$ from the center of the Cylinder [Albella et al., 2007].

near-field plot of the perfect cylinder and will be used as a reference. Figure 7(b) and 7(c) correspond to the cases of a metallic defect on the cylinder. The outline of the defect is visible on these panels. It can be seen that the defect does not change significantly the shape of the near field when compared to the perfect cylinder case. Figure 7(d) and 7(e) correspond to the cases of a metallic defect on the substrate. In Figure 7(d), the defect is farthest from the cylinder, outside the shadow region, and we observe a significantly different field distribution around the micron-sized particle located in what initially was a maximum of the local field produced by the main cylinder. The same feature can be found in the far-field plot. This case corresponds to the maximum change with respect to the nondefect case. Finally, Figure 7(e) corresponds to the case of a metallic defect on the substrate and close to the cylinder, very close to the position shown in Figure 7(c). As expected, both cases are almost indistinguishable. 
(a)

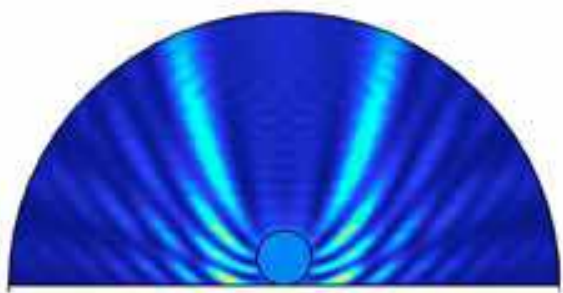

Perfect Cylinder

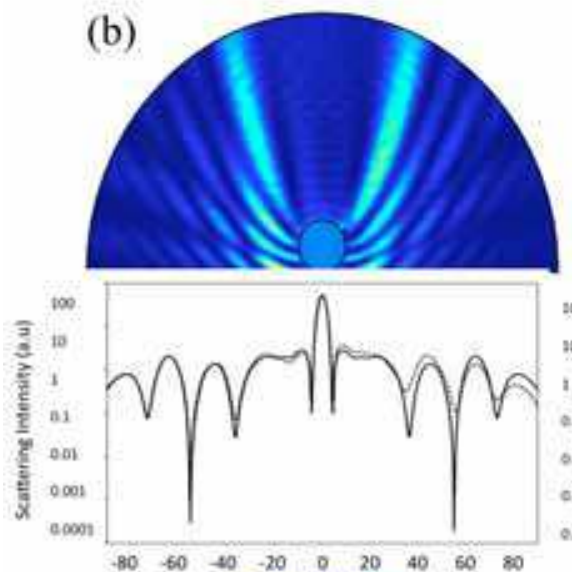

(d)

(c)
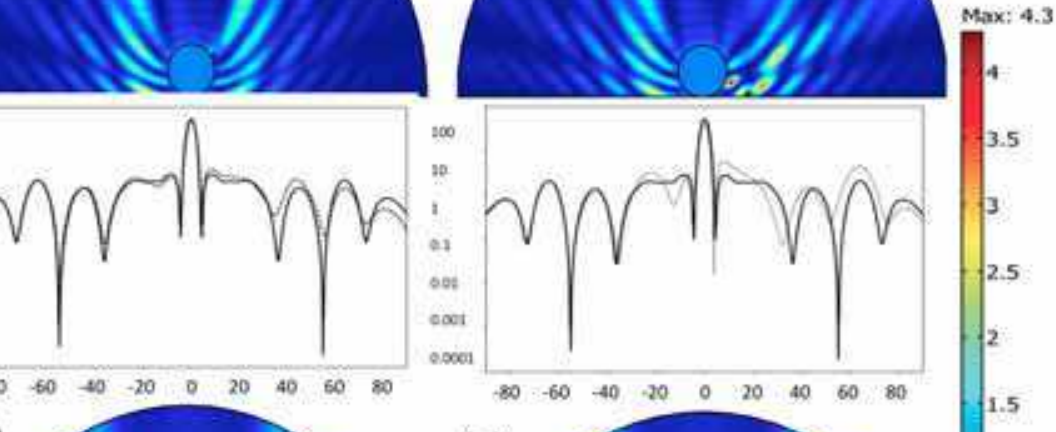

(e)
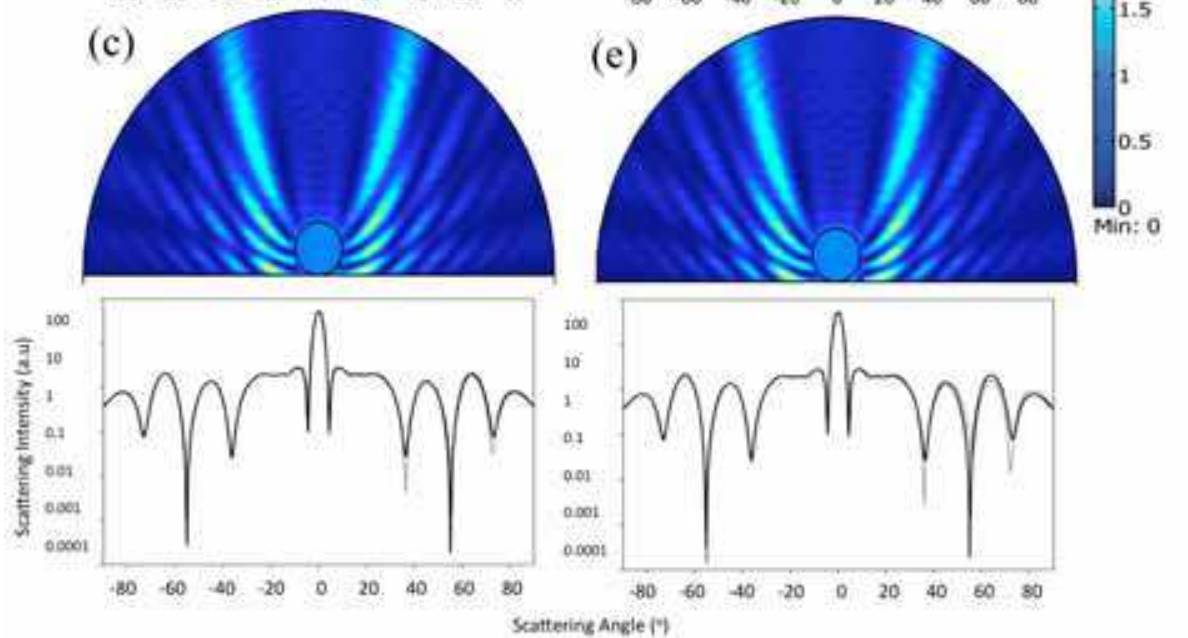

Fig. 7. (a) Near field plots for a silver cylinder sized $D=2 \lambda$ located on a silver substrate and illuminated at normal incidence. (b) and (c) show patterns for the defect located on the cylinder and (d) and (e) show patterns for the defect located on the substrate. The main cylinder and defect are outlined in black. Each plot has its corresponding far-field at the bottom compared to the NPC case pattern. 


\section{Influence of the optical properties of the substrate}

In this section, we discuss the sensitivity of the defect detection technique to the optical properties of the substrate in the two possible situations described before: (A) with the defect on the cylinder and (B) with the defect on the substrate near the cylinder. We will see how the substrate can affect the detection capabilities in each particular situation.

In the previous sections, we described how a small defect located on a micron-sized silver cylinder on a substrate changes the backscattered intensity. We showed that an integration of the backscattered intensity over either the positive or negative quadrant, corresponding to the defect side or the opposite side, yields a parameter $\sigma_{b r}$ sensitive not only to the existence of the defect but also to its size and location on the microstructure. These results were initially obtained for perfectly conducting systems and later on, for more realistic systems: dielectric or metallic defects on a metallic cylinder located on a metallic substrate. From a practical point of view, detection and sizing of very small defects on microstructures located on any kind of substrate by non-invasive methods could be very useful in qualitycontrol technology and in nano-scale monitoring processes. This section is focused on examining the sensitivity of this technique to the optical properties of the substrate in the aforementioned situations. In this section we also consider the cylinder to be composed of gold.

\subsection{Defect on the cylinder}

Figure 8 shows a comparison between the backscattered intensity pattern for a perturbed gold cylinder located on a metallic gold substrate and the backscattering pattern obtained for the same system located on other substrates having different optical properties. The diameter of the main cylinder and of the defect are $D=\lambda$ and $d=0.1 \lambda$, respectively, thus keeping constant the ratio $d / D=0.1$. The defect position has been fixed on the cylinder at $\varphi=$ $50^{\circ}$ as it is one of the most representative cases.

As can be observed for backscattering angles $\theta_{s}<0$, that is when the cylinder shadows the defect, the shape of the pattern remains essentially the same. We also do see slightly smaller values as we increase the dielectric constant of the substrate. When we illuminate the system on the same side as the defect, $\theta_{s}>0$, we tend to see the opposite behaviour: in the locations where the values of $I_{\text {back }}$ increased as we increase the dielectric constant of the substrate, and approaching the values of $I_{b a c k}$ for the metallic substrate case. Although the changes in the backscattering induced by the defect may seem negligible, we will see that these differences can be monitored with appropriate integrating parameters. In particular, we use the integrated backscattering parameter $\sigma_{b r}$ as defined in the previous sections.

Figure 9(a) shows the behaviour of $\sigma_{b r}$ for different dielectric substrates as a function of the angular position of the defect. An interesting result is the increase of $\left|\sigma_{b r}{ }^{+}\right|$as we increase $\varepsilon$, reaching a maximum for the case of a metal. The opposite behaviour is observed for $\left|\sigma_{b r}{ }^{-}\right|$. The maximum absolute value of $\sigma_{b r}{ }^{+}$and $\sigma_{b r}{ }^{-}$for pure dielectric substrates is plotted in Figure $9(b)$ as a function of the substrate dielectric constant $\varepsilon$. We notice that the quantity $\sigma_{b r}$ is more sensitive to $\varepsilon$ within the interval $\varepsilon \in[1.2,4]$ and it saturates for high values of $\varepsilon$, tending to the metal substrate case. Absorption has not been considered for the case of real dielectrics as it is very small in the visible range. Another interesting result is that for a defect on the upper part of the cylinder $\varphi<50^{\circ}, \sigma_{b r}{ }^{-}$is very sensitive to the metal/dielectric nature of the substrate. On the other hand, these remarkable values of $\sigma_{b r}{ }^{-}$make it more difficult to locate the position of the defect. 

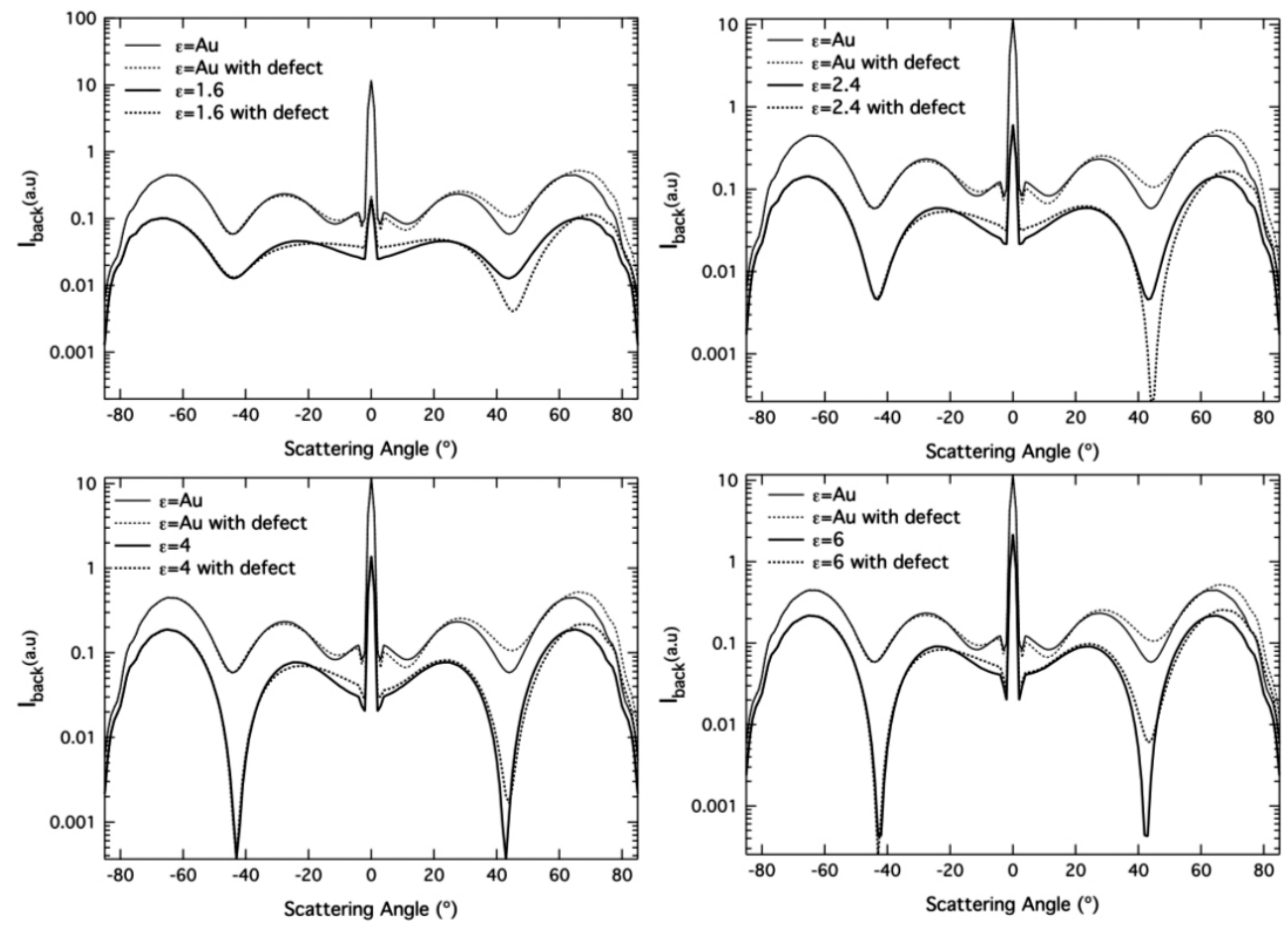

Fig. 8. Backscattering patterns for a defect on a cylinder placed on a substrate for different substrate optical properties $(\varepsilon)$. The defect and cylinder are made of gold and of size $d=0.1 \lambda$ and $D=\lambda$ respectively.

(a)

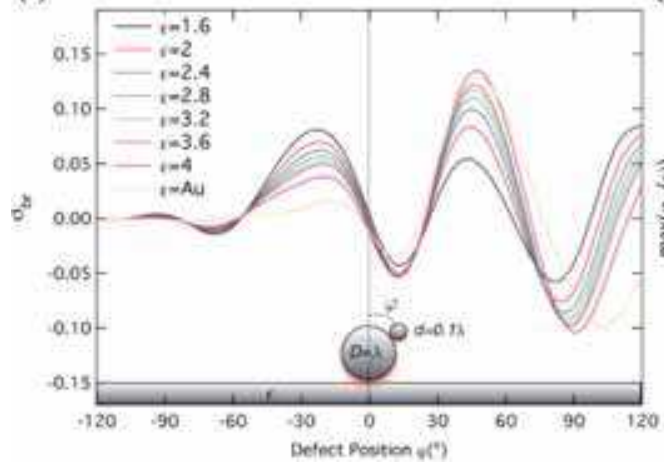

(b)

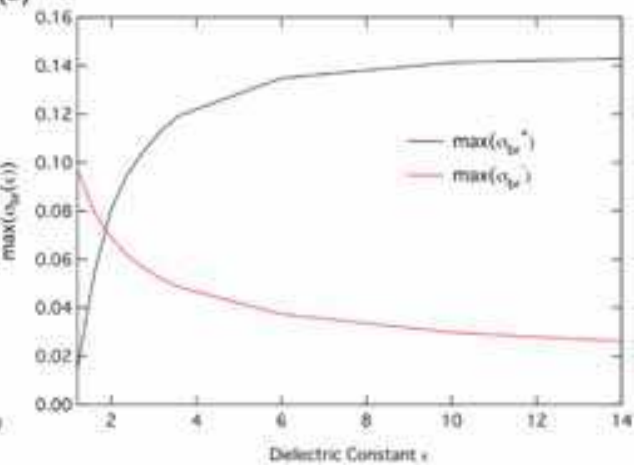

Fig. 9. (a) Evolution of $\sigma_{b r}$ for a defect on a cylinder as a function of the optical properties of the substrate. (b) Evolution of $\max \left(\sigma^{+}\right)$and $\max \left(\sigma^{-}\right)$for pure dielectric substrates as a function of the substrate dielectric constant, $\varepsilon$ [Albella et al., 2008]. 


\subsection{Defect on the substrate}

Figure 10 shows a series of graphs comparing the patterns obtained for different dielectric substrates with those obtained for a metallic substrate for a fixed defect position $x=3 \lambda / 4$. For $\theta_{s}<0$, i.e. the region opposite to the defect side, the change induced by the defect in the backscattering pattern is not significant, independent of the kind of substrate material. However, when $\theta_{s}>0$, the backscattering is strongly affected by the defect, especially for the dielectric substrate. For increasing values of the dielectric constant, the change induced by the defect becomes smaller. This is the opposite of what was found when the defect was on the cylinder.
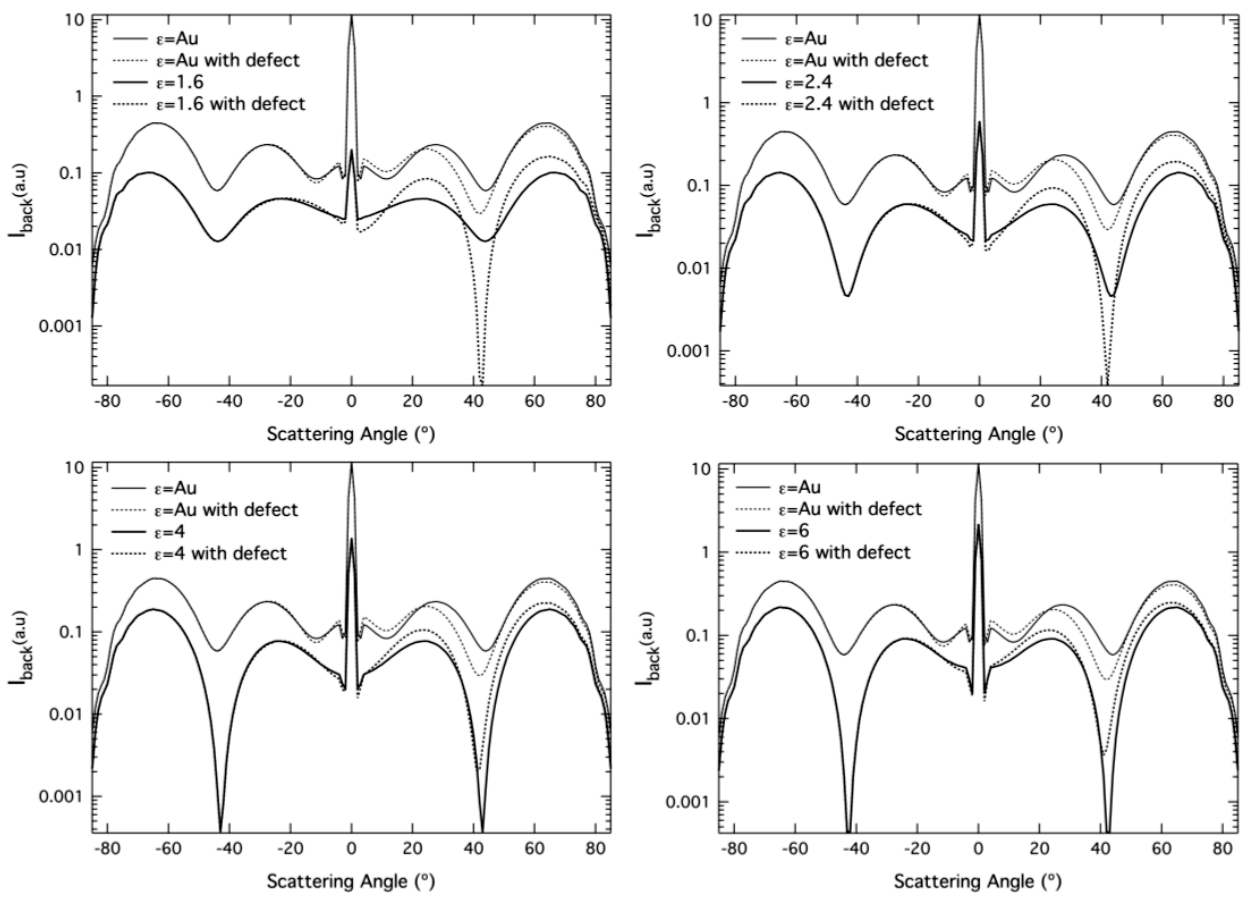

Fig. 10. The backscattered intensity pattern $I_{b a c k}$ as a function of the incident angle $\theta_{i}$.

Parameter $\sigma_{b r}$ allows for a straightforward assessment of these defects. Figure 11 shows the evolution of $\sigma_{b r}$ for different dielectric and metallic substrates as a function of the position $x$ of the defect in the substrate. The shadowed area represents defect positions under the cylinder, not considered in the calculations. The most interesting feature of the curves shown in Figure 11(a) is the high sensitivity of $\left|\sigma_{b r}{ }^{+}\right|$to the presence of a defect for the case of dielectric substrates. This sensitivity grows when the contrast in refractive index between the defect and the substrate increases. The maxima of $\sigma_{b r}{ }^{+}$and $\sigma_{b r}{ }^{-}$are plotted in Figure 11(b) as a function of $\varepsilon$ for the dielectric substrate case. Both decrease and saturate for large values of $\varepsilon$. It is also worth remarking that when the defect is on the substrate, $\sigma_{b r}>0$, except for some positions corresponding to the metallic substrate case. This means that, on average, the backscattering is enhanced by a particle on the dielectric substrate, but it can be reduced when the defect lies on a metallic substrate. However, when the defect is on the cylinder, negative values are found for either kind of substrate. 
(a)

(b)
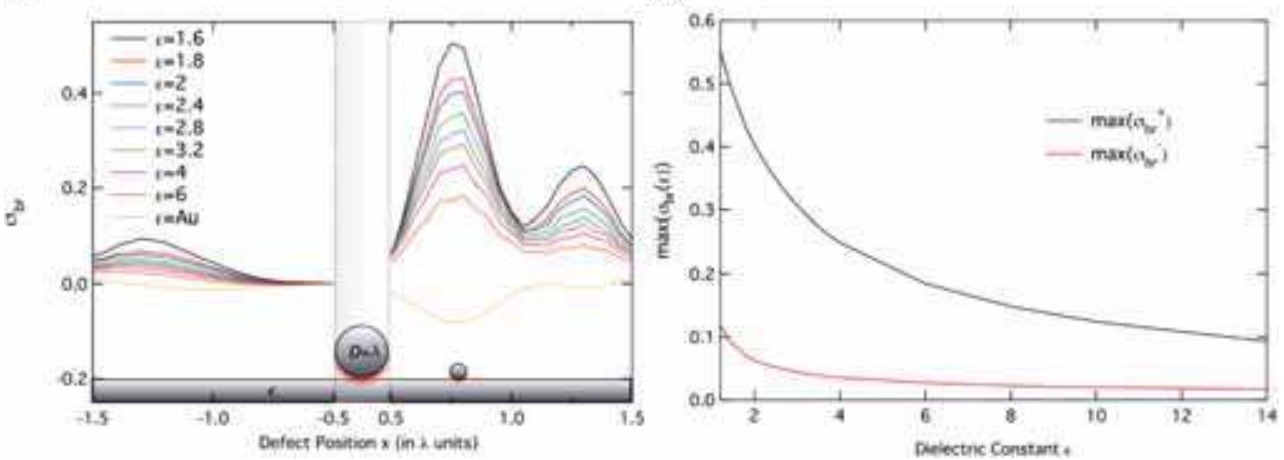

Fig. 11. (a) Evolution of $\sigma_{b r}$ as a function of the substrate optical properties. A defect lies on the substrate. (b) Evolution of $\max \left(\sigma^{+}\right)$and $\max \left(\sigma^{-}\right)$for pure dielectric substrates as a function of the substrate dielectric constant $\varepsilon$ [Albella et al., 2008].
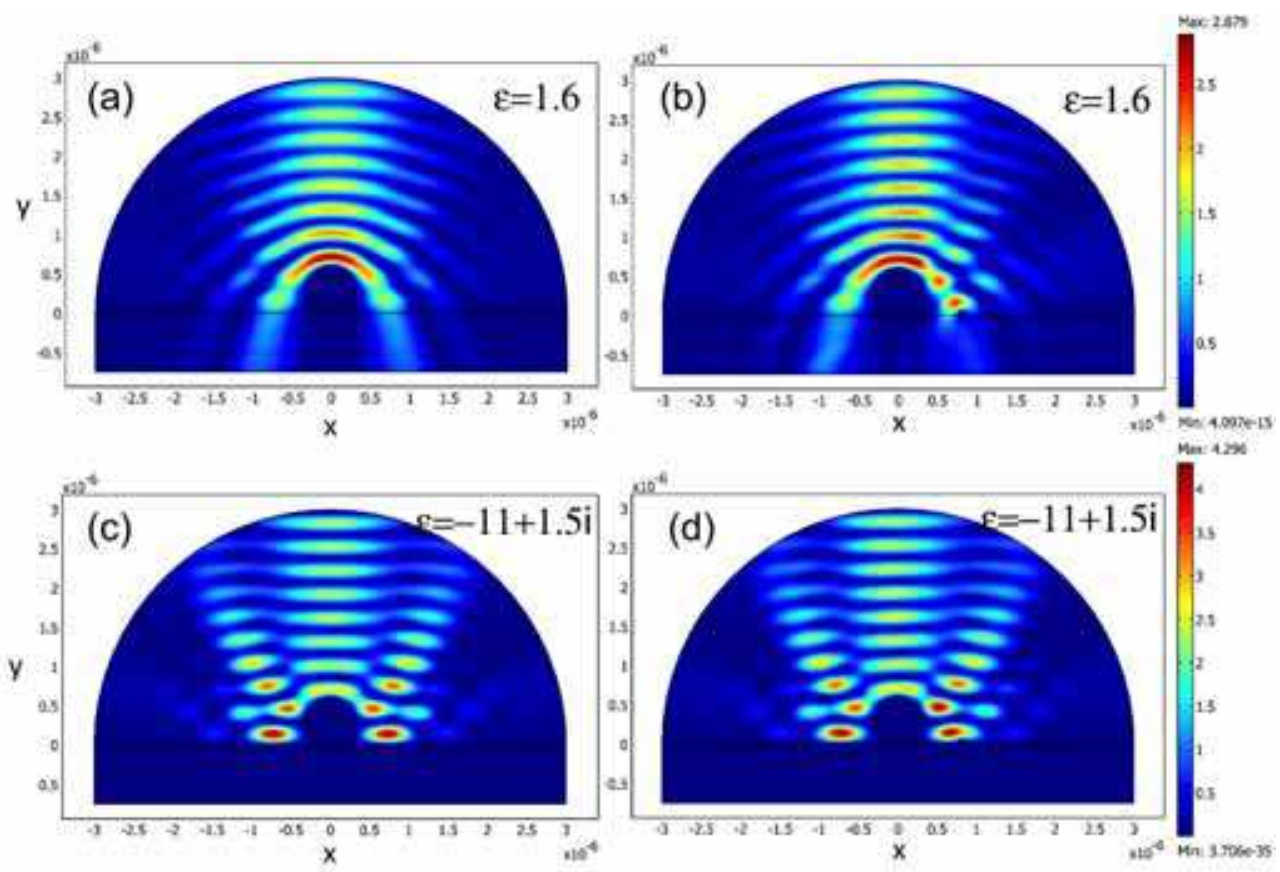

Fig. 12. Near-field plots corresponding to two different substrates illuminated at normal incidence. The figures on the left correspond to the reference case having no defect and on the right to a defect on the substrate. On the top are results for a dielectric substrate $\varepsilon=1.6$ and on the bottom for a gold substrate $\varepsilon=11+1.5 i$.

Finally, to illustrate this enhancement, Figure 12 shows some examples of the near-field pattern obtained for two different substrates illuminated at normal incidence. The figures on 
the left correspond to the reference case having no defect and on the right to a defect on the substrate. On the top are results for a dielectric substrate $\varepsilon=1.6$ and on the bottom for a gold substrate. We see that for low values of $\varepsilon$, we observe a new spatial distribution around the micron-sized particle resulting in a new local maximum of intensity, which produces the maximum change with respect to the non-defect case. This strong change in the near-field distribution is not surprising since this defect position corresponds to a maximum in $\sigma_{b r}{ }^{+}$as observed in Figure 11(b). Near-field plots (Figure 12) show a close correlation among the maxima located near the microstructure and the angular $(\varphi)$ and linear $(x)$ positions of the maxima obtained for $\sigma_{b r}$ plots.

\section{Conclusion}

The results in this chapter suggest that the measurement of $\sigma_{b r}$ can be a useful means of monitoring, sizing and characterizing small defects adhered to microstructures or substrates. From a practical point of view, detection and sizing of very small defects on microstructures by some reliable and non-invasive method is useful in quality control technology. In this context, the objective was to study the sensitivity of this technique to the optical properties of the substrate in two situations: (A) when the defect was located on a cylinder and (B) when the defect was located on the substrate near a cylinder. We also have anticipated how choosing the appropriate material for the substrate in each particular situation can influence this detection. In this chapter a system structured at two different levels has been analyzed by studying its scattering properties in the backscattering direction.

A parameter $\sigma_{b}^{ \pm}$defined as the integration of the backscattered intensity over a given quadrant is a quantitative measure of overall backscattering variations. It can be used, for instance, by measuring the relative variation with respect to an initial system $\sigma_{b r}{ }^{ \pm}$as has been discussed in this chapter, as a simple measure of asymmetry through $\left|\sigma_{b}{ }^{+}-\sigma_{b}{ }^{-}\right|$, or through a backscattering asymmetry index $2\left|\sigma_{b}{ }^{-}-\sigma_{b}-\right| /\left(\sigma_{b}{ }^{-}-\sigma_{b}{ }^{-}\right)$, presumably suitable for experimental situations. Parameter $\sigma_{b}$ itself can be experimentally obtained through different configurations [Peña et al., 2000].

The potential uses of a parameter like $\sigma_{b r}{ }^{ \pm}$depend very much on the needs of the research and on any previous knowledge. When applied to the double-cylinder case, the following has been demonstrated:

i. With the defect on the cylinder, parameter $\sigma_{b r}{ }^{ \pm}$depends on the defect size and position, while when the defect is on the substrate, $\sigma_{b r} \pm$ is also dependent on the main particle size $D$;

ii. With the defect on the cylinder, its composition (metal/dielectric) may be identified from the sign of $\sigma_{b r}{ }^{ \pm}$for a given defect position;

iii. A remarkable increase of $\sigma_{b r}{ }^{-}$is characteristic of the defect being on the substrate and is not found when the defect is on the principle particle;

iv. Strong oscillations in $\sigma_{b r}{ }^{+}$observed when the defect is on the substrate for small values of $x$ can identify very precisely the position of the defect;

v. The use of metallic or dielectric substrates does not affect significantly the behaviour of $\sigma_{b r}$ and therefore does not present a limitation for this parameter.

This is not the first time that changes in the backscattering pattern obtained from a simple configuration allows for the characterization of some geometrical or material changes produced in the scattering object, but we think the procedure shown here is applicable to a 
wide range of $2 \mathrm{D}$ structures whose defects often require rapid identification. Finally, these results have a natural extension to $3 \mathrm{D}$ geometries that would enlarge the range of practical situations and the scope of this work. This would require more powerful computing techniques and tools applied over an extensive caustic. Everything shown in this chapter can be seen as the basis for analyzing more complex geometrical systems.

\section{Appendix}

A particle sizing method is proposed using a double-interaction model (DIM) for the light scattered by particles on substrates. This model, based on that proposed by Nahm and Wolfe [Nahm \& Wolfe, 1987], accounts for the reflection of both the incident and the scattered beams and reproduces the scattering patterns produced by particles on substrates, provided that the angle of incidence, the polarization and the isolated particle scattering pattern are known.

In this context, we show the results obtained using a simple model to assess the effect of the presence of a nano-defect on a microstructure located on a substrate. This three-object system can be modeled with an extension of the double interaction model, which was shown to be useful for obtaining the electric field scattered from a single particle resting on a substrate. In this chapter, we extend that model in a 2D frame, to a system where there are two metallic cylinders, one being a nano-defect lying on a micron-sized structure that rests on a flat substrate. We also show how this simple model reproduces the scattering pattern variations with respect to the defect-free system when compared to that given by an exact method. It is important to remark that this model has two interesting features: (1) Transparency, in that it is easy to understand the mechanisms involved in the scattering; and (2) Easy numerical implementation that can lead to fast computation.

\section{Model description}

The system consists of an infinitely long metallic cylinder of radius $R$ placed on a flat substrate (see Figure 13) while another, much smaller, cylinder rests on top of the first. Both cylinders are assumed metallic, and in our calculations we give them the properties of silver. The cylinder axis is parallel to the $\mathrm{Y}$ direction, and the $\mathrm{X}-\mathrm{Z}$ plane is the scattering plane. This reduces the geometry to $2 \mathrm{D}$. The model we propose in this work can be described as an application of the DIM [Nahm \& Wolfe, 1987] for normal incidence in two steps: (1) to the large cylindrical microstructure located on the flat substrate and (2) to the small cylindrical nano-defect, assuming that the underlying cylinder approximates a flat substrate for the small cylinder. Of course, the accuracy of this second approximation improves as the ratio $r / R$ approaches zero. We shall limit our solution to the range $0<r / R<0.1$.

The DIM is based on the standard T-matrix solution for the isolated scatterer at normal incidence. Two contributions to the total scattered field are generated in any direction: the light directly scattered from the particle, and that scattered and reflected off the substrate. The latter is affected by a complex Fresnel coefficient, thus having its phase shifted because of its additional path.

If we now consider the two-particle system, the total scattered far field at a fixed scattering direction given by the scattering angle $\theta_{s}$ is the coherent superposition of four contributions, two of them due to the large cylinder and two due to the small one. Looking at Figure 13, arrows labelled (1) and (3) represent the components directly scattered by the particles to 


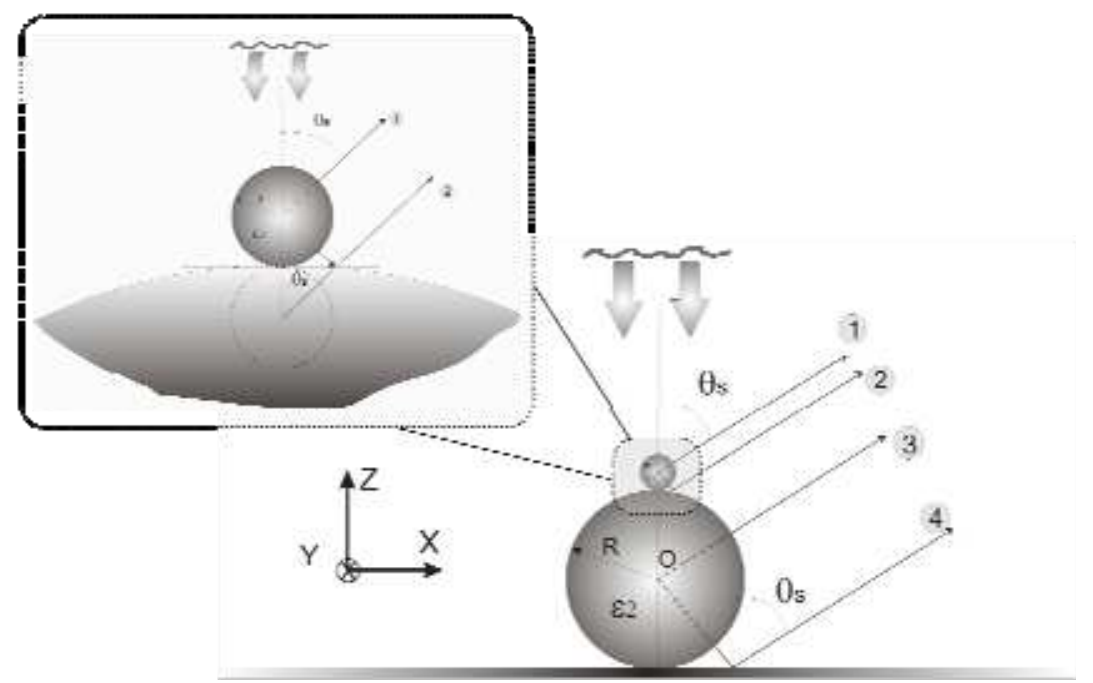

Fig. 13. Multiple interaction model for two structures illuminated at normal incidence. Inset shows the flat substrate approximation and image theory applied to the defect.

the detector; and arrows labelled (2) and (4) correspond to the components scattered downwards by the particles and then reflected towards the observation angle.

Simple calculations allow us to account for the phase shifts produced by the extra-path of each contribution from a plane normal to the incidence direction to a plane normal to the observation. Taking the direct contribution (3) as the reference beam having zero phase $\left(\delta_{3}=0\right)$ :

$$
\begin{gathered}
\delta_{1}\left(\theta_{s}\right)=-\frac{2 \pi(R+r) \cdot\left(1+\cos \theta_{s}\right)}{\lambda} \\
\delta_{2}\left(\theta_{s}\right)=\delta_{1}+\frac{4 \pi \cdot r \cos \theta_{s}}{\lambda} \\
\delta_{4}\left(\theta_{s}\right)=\frac{4 \pi \cdot R \cos \theta_{s}}{\lambda}
\end{gathered}
$$

where $\theta_{s}$ is the scattering angle, $R$ and $r$ are the radii of the large and small cylinders respectively, and $\lambda$ is the incident wavelength. Reflection from the substrate is simplified using the plane wave approximation and considering S-polarization, and is given by the Fresnel reflection coefficient in its complex form,

$$
\hat{r}_{s}(\theta)=\frac{\cos \theta-\sqrt{\hat{\varepsilon}_{\text {sub }}-\sin ^{2}(\theta)}}{\cos \theta+\sqrt{\hat{\varepsilon}_{\text {sub }}-\sin ^{2}(\theta)}}
$$

The total scattered electric field under the assumptions of this Combined Double Interaction Model (CDIM) is given by the sum of the four contributions, 


$$
\begin{gathered}
E_{1}\left(\theta_{s}\right)=A_{0} \cdot F\left(180^{o}-\theta_{s}\right) \cdot e^{i \cdot\left(\phi_{1}+\delta_{1}\right)} \\
E_{2}\left(\theta_{s}\right)=A_{0} \cdot F\left(\theta_{s}\right) \cdot\left|r\left(\theta_{s}\right)\right| \cdot e^{i \cdot\left(\phi_{2}+\delta_{2}+\alpha_{s}\right)} \\
E_{3}\left(\theta_{s}\right)=A_{0} \cdot F\left(180^{o}-\theta_{s}\right) \cdot e^{i \cdot \phi_{3}} \\
E_{4}\left(\theta_{s}\right)=A_{0} \cdot F\left(\theta_{s}\right) \cdot\left|r\left(\theta_{s}\right)\right| \cdot e^{i \cdot\left(\phi_{4}+\delta_{4}+\alpha_{s}\right)}
\end{gathered}
$$

where $A_{0}$ is the amplitude of the incident beam, and the complex terms have been expressed in their polar form, $\varphi_{i}$ is the Mie phase corresponding to the $i$ th contribution, $F\left(\theta_{s}\right)$ is the amplitude of the scattered electric far field in the $\theta_{s}$ direction for an isolated particle, which are given by the $T$-matrix amplitudes applied to the case of a cylinder, and $a_{s}$ is the phase introduced by the Fresnel reflection.

\section{Testing the model}

Results obtained by using the CDIM are compared with others obtained from the ET method, which is an exact rendering of the Maxwell equations in their integral form. Figure 14 shows the scattering intensity patterns obtained from the CDIM approximation (top) and from the ET (bottom) in semi-logarithmic scale. CDIM results have been shifted upwards for an easy visualization. The continuous line plots correspond to the defect-free situation (single cylinder) in all cases, while the dashed line corresponds to the case of a defect located on the top of the cylinder.

In Figure 15 we consider the range of validity of this model. CDIM and ET are compared for $R=\lambda$ and for $r=0.05 \lambda, 0.1 \lambda$ and $0.15 \lambda$. We observe that both patterns show the same outer minima positions for a defect/cylinder aspect ratio $(r / R)$ up to 0.1 , while for the case $r / R=$ 0.15 , distortions in the pattern become important. The CDIM reproduces the changes produced in the lobed structure of the scattering patterns and particularly the minima positions. If we look at Figure 14(a) as an example, we can see that in the case of the exact solution (shown at the bottom part of the graph), there is a shift in the minima produced by the presence of the defect, and this shift is accurately reproduced by the model.

One limit imposed on the ratio $r / R$ is due to assuming the underlying main cylinder is flat. For values of $r / R<0.1$ the changes introduced by the real curvature in the Fresnel coefficients and in the T-matrix scattering amplitude are very small and the changes in the phase of the contribution due to the optical path difference are negligible as long as $R$ is of the order of the wavelength.

Although this problem may be overcome by numerically calculating the exact angular reflections and paths, it is worthwhile to consider this model for its simplicity and transparency. Because of its simplicity, the model can be extended to other situations, for instance, a 3D implementation by introducing the Mie coefficients for spheres, different contaminating particles, different defect locations, etc.

\section{Acknowledgements}

The authors wish to acknowledge the funds provided by the Ministry of Education of Spain under project \#FIS2007-60158. We also thank the computer resources provided by the Spanish Supercomputing Network (RES) node at Universidad de Cantabria. 

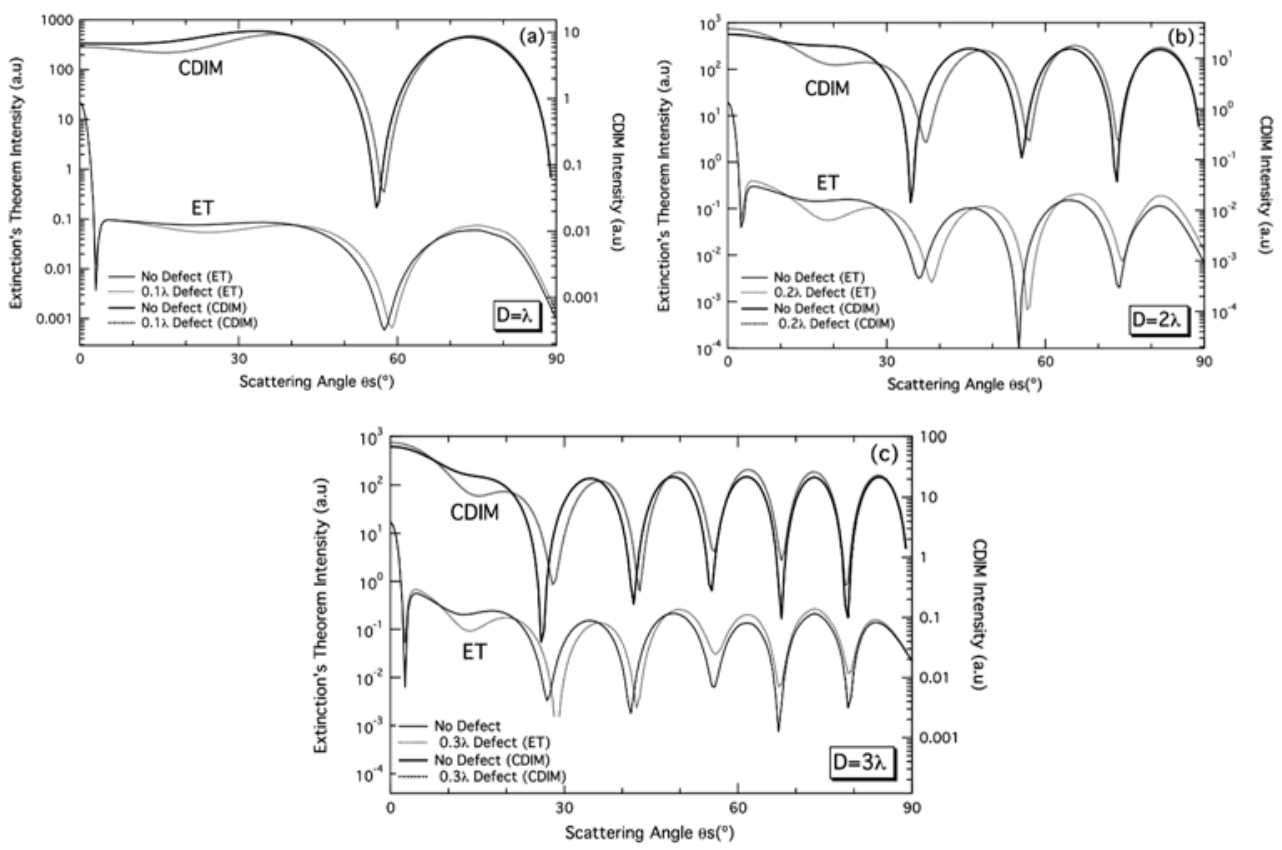

Fig. 14. Scattering patterns calculated using CDIM and ET for three different sizes of the underlying cylinder, keeping a constant defect/cylinder aspect ratio of 0.1 : (a). $R=\lambda / 2$ and $r$ $=\lambda / 20 ;(\mathrm{b}) . R=\lambda$ and $r=\lambda / 10 ;$ (c). $R=1.5 \lambda$ and $r=0.15 \lambda$. This corresponds with defect sizes of 60, 120 and $180 \mathrm{~nm}$ for an incident wavelength of $0.6 \mu \mathrm{m}$ [Albella et al., 2007].

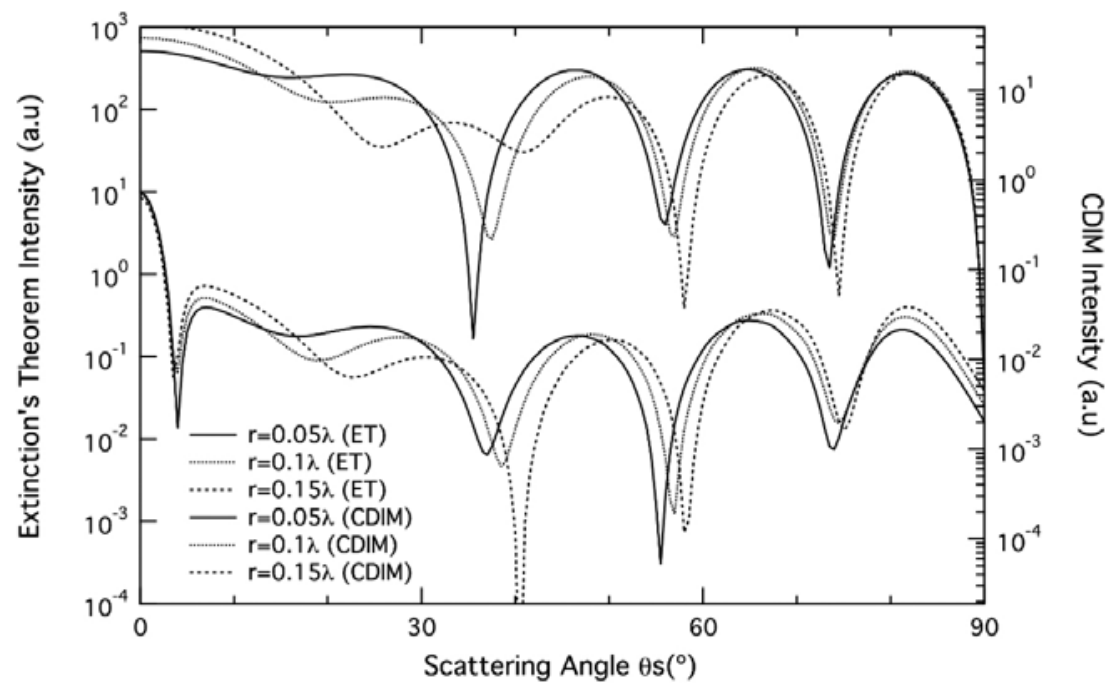

Fig. 15. Scattering pattern of an $R=\lambda$ cylinder with a defect of $r=0.05 \lambda, 0.1 \lambda$ and $0.15 \lambda$ calculated using CDIM (top) and ET (bottom) [Albella et al., 2007]. 


\section{References}

Albella, P., F. Moreno, J. M. Saiz, and F. González, "Monitoring small defects on surface microstructures through backscattering measurements." Opt. Lett. 31, 1744-1746, (2006).

Albella, P., F. Moreno, J. M. Saiz, and F. González, "Backscattering of metallic microstructures will small defects located on flat substrates." Opt. Exp. 15, (2007) 6857-6867.

Albella, P., F. Moreno, J. M. Saiz, and F. González, "2D double interaction method for modeling small particles contaminating microstructures located on substrates." J. Quant. Spectrosc. Radiative Trans. 106, 4-10 (2007).

Albella, P., F. Moreno, J. M. Saiz, and F. González, "Influence of the Substrate Optical Properties on the backscattering of contaminated microstructures." J. Quant. Spectrosc. Radiative Trans. 109, (2008) 1339-1346.

Chen, H. T., R. Kersting and G. C. Cho, "Terahertz imaging with nanometer resolution." Appl. Phys. Lett. 83, (2003) 3.

Germer, T. A., and G. W. Mulholland. "Size metrology comparison between aerosol electrical mobility and laser surface light scattering," Characterization and Metrology for ULSI Technology, (2005), 579-583.

Germer, T. A., “Light scattering by slightly non-spherical particles on surfaces," Opt. Lett. 27, 1159-1161 (2002).

Johnson, B. R, "Light scattering from a spherical particle on a conducting plane, in normal incidence," J. Opt. Soc. Am. A 19, 11 (2002).

Lee, K. G., H. W. Kihm, J. E. Kihm, W. J. Choi, H. Kim, C. Ropers, D. J. Park, Y. C. Yoon, S. B. Choi, D. H. Woo, J. Kim, B. Lee, Q. H. Park, C. Lienau, and D. S. Kim, "Vector field microscopic imaging of light," Nature Photonics 1, (2007) 53-56.

Liswith, M. L., E. J. Bawolek, and E. D. Hirleman, "Modeling of light scattering by submicrometer spherical particles on silicon and oxidized silicon surfaces," Opt. Eng. 35, 858-869 (1996).

Mittal, K. L. (editor). Particles on surfaces: Detection, Adhesion and Removal. VSP, Utrech (1999).

Moreno, F and F. González Eds. Light scattering from microstructures. Springer Verlag (2000).

Moreno, F., F. González, and J. M. Saiz, "Plasmon spectroscopy of metallic nanoparticles above flat dielectric substrates," Opt. Lett., 31, (2006), 1902-1904.

Mulholland, G. W, T. A. Germer, and J. C. Stover, "Modeling, measurement and standards for wafer inspection," Proceedings of the Government Microcircuits Applications and Critical Technologies. (2003), 1-4.

Nahm, K. B., and W. L. Wolfe, "Light-scattering models for spheres on a conducting plane," Appl. Opt. 26, (1987), 2995-2999.

Nieto-Vesperinas, M., and J. A. Sánchez-Gil, "Light scattering from a random rough interface with total internal reflection," J. Opt. Soc. Am. A 9, (1992) 424-436.

Peña, J.L., J. M. Saiz, P. Valle, F. González, and F. Moreno, “Tracking scattering minima to size metallic particles on flat substrates," Particle $\mathcal{E}$ Particle Systems Characterization 16, (1999) 113-118.

Ripoll, J., A. Madrazo, and M. Nieto-Vesperinas, "Scattering of electromagnetic waves from a body over a random rough surface," Opt. Comm. 142, (1997) 173-178. 
Saiz, J.M., P. J. Valle, F. González, E. M. Ortiz, and F. Moreno, "Scattering by a metallic cylinder on a substrate: burying effects," Opt. Lett., 21, (1996) 1330-1332.

Sánchez-Gil, J. A., and M. Nieto-Vesperinas, "Resonance effects in multiple light scattering from statistically rough metallic surfaces," Phys. Rev. B 45 8623-8633 (1992).

Sonnichsen, C., B. M. Reinhard, J. Liphardt, and A. P. Alivisatos, "A molecular ruler based on plasmon coupling of single gold and silver nanoparticles," Nature Biotechnology 23, (2005), 741-745.

Stuart, D., A. J. Haes, C. R. Yonzon, E. Hicks, and R. V. Duyne, "Biological applications of localised surface plasmonic phenomenae," IEE Proc., Nanobiotechnology 152, (2005) 13-32.

Valle, P., F. González, and F. Moreno, "Electromagnetic wave scattering from conducting cylindrical structures on flat substrates: study by means of the extinction theorem," Appl. Opt. 33, (1994), 512-523. 


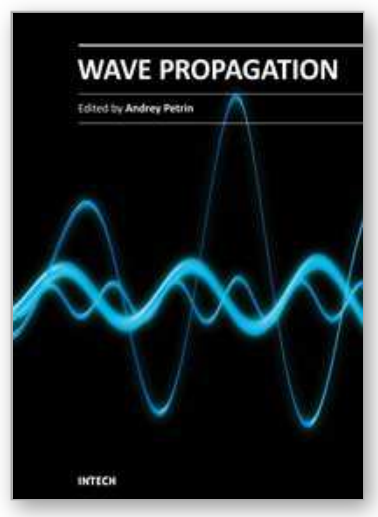

\author{
Wave Propagation \\ Edited by Dr. Andrey Petrin
}

ISBN 978-953-307-275-3

Hard cover, 570 pages

Publisher InTech

Published online 16, March, 2011

Published in print edition March, 2011

The book collects original and innovative research studies of the experienced and actively working scientists in the field of wave propagation which produced new methods in this area of research and obtained new and important results. Every chapter of this book is the result of the authors achieved in the particular field of research. The themes of the studies vary from investigation on modern applications such as metamaterials, photonic crystals and nanofocusing of light to the traditional engineering applications of electrodynamics such as antennas, waveguides and radar investigations.

\title{
How to reference
}

In order to correctly reference this scholarly work, feel free to copy and paste the following:

Pablo Albella, Francisco González, Fernando Moreno, José María Saiz and Gorden Videen (2011). Detection and Characterization of Nano-Defects Located on Micro-Structured Substrates by Means of Light Scattering, Wave Propagation, Dr. Andrey Petrin (Ed.), ISBN: 978-953-307-275-3, InTech, Available from: http://www.intechopen.com/books/wave-propagation/detection-and-characterization-of-nano-defects-locatedon-micro-structured-substrates-by-means-of-li

\section{INTECH}

open science | open minds

\section{InTech Europe}

University Campus STeP Ri

Slavka Krautzeka 83/A

51000 Rijeka, Croatia

Phone: +385 (51) 770447

Fax: +385 (51) 686166

www.intechopen.com

\section{InTech China}

Unit 405, Office Block, Hotel Equatorial Shanghai

No.65, Yan An Road (West), Shanghai, 200040, China

中国上海市延安西路65号上海国际贵都大饭店办公楼405单元

Phone: +86-21-62489820

Fax: $+86-21-62489821$ 
(C) 2011 The Author(s). Licensee IntechOpen. This chapter is distributed under the terms of the Creative Commons Attribution-NonCommercialShareAlike-3.0 License, which permits use, distribution and reproduction for non-commercial purposes, provided the original is properly cited and derivative works building on this content are distributed under the same license. 